\title{
GRDG Analysis of the ALSTOM Gasifier Benchmark Process under Model Plant Mismatch
}

\author{
Rudy Agustriyanto ${ }^{1,2}$ and Jie Zhang ${ }^{1}$ \\ ${ }^{1}$ School of Chemical Engineering and Advanced Material, Newcastle University, Newcastle upon Tyne, NE1 7RU, UK \\ (Tel : +44-191-2227240; E-mail: rudy.agustriyanto@newcast le.ac.uk; ji ie.zhang@newcast le.ac.uk) \\ ${ }^{2}$ Department of Chemical Engineering, Surabaya University (UBAYA), Surabaya, Indonesia
}

\begin{abstract}
An optimization based method for determining relative disturbance gain array (RDGA) range for uncertain system is presented and is then applied to control structure analysis of the ALSTOM gasifier benchmark process. Reduced order linear models were identified from simulated plant operating data. Several different models were obtained under different operating conditions and model uncertainty bounds were obtained from those models. The generalized relative disturbance gain (GRDG) ranges under plant model uncertainty were then determined from a structure selection matrix in conjunction with relative disturbance gain array (RDGA) range and used in selecting robust decentralized control structure. GRDG bounds are particularly useful for control structure determination and the related robustness as they provide information regarding the sensitivity to gain uncertainties.
\end{abstract}

Keywords: Process control, robust control, uncertain systems.

\section{INTRODUCTION}

A typical complex chemical plant may have hundreds of measurements and control loops. According to Luyben (1988), plantwide control involves the systems and strategies required to control an entire chemical plant consisting of many interconnected unit operations, or in other words, Skogestad (2004) described plantwide control as control structure design for complete chemical plants. Commonly, plantwide control concerns the control philosophy of the overall plant with emphasis on control structure determinations, which includes the selection and pairing of controlled and manipulated variables as well as decomposition of the overall problem into smaller sub problems (the control configuration). The objectives of control structure determination are usually described in terms of 'process operability, 'process controllability', 'process stability' etc.

Generalized relative disturbance gain (GRDG) is one of the steady state techniques for process operability analysis and has been widely used in process control to evaluate the load effect under a specific controller structure (Chang and $\mathrm{Yu}, 1992$ ), hence it is useful to identify promising control structures. In GRDG analysis, sensitivity of the GRDG subject to model uncertainty is important since process models are never perfect. An analysis based on nominal process and disturbance models can lead to incorrect conclusions for uncertain process and disturbance models.

Recently Chen and Seborg (2002) presented analytical expressions for relative gain array (RGA) uncertainty bounds where all elements of the steady state process gain matrix are allowed to change simultaneously. A different method using the structured singular value $(\mu)$ framework was also introduced for calculation of the magnitude of the worst case relative gain (Kariwala et al., 2006), but so far unfortunately less attention had been given for relative disturbance gain (RDG).
In 1997, ALSTOM Power Technology issued an open challenge to the UK academic control community, which addressed the control of a gasifier plant (Dixon et al., 2000). The 'challenge information pack' included three linear models (obtained from ALSTOM's comprehensive non-linear model of the plant). Full detail of this challenge can be found in references (Burnham et al., 2000; Dixon et al., 2000).

Among the approaches that have been proposed to solve this challenging problem, Asmar et al. (2000) provide a relatively simple controller structure but with excellent performance. It only fails in its regulation task during one of the six pressure disturbance tests. Later this structure was adopted and used as a baseline controller in the second round of ALSTOM benchmark challenge (Dixon and Pike, 2004), where the nonlinear simulations programme for the process is provided. However, detailed analysis on why this baseline control structure performed well has not been reported.

This paper presents an optimization based method for calculating GRDG ranges under model uncertainty. The method is applied to the ALSTOM gasifier benchmark process in order to select the robust control structure. Simulation results confirm the theoretical analysis.

\section{THE ALSTOM GASIFER BENCHMARK PROBLEM}

\subsection{The Process}

The ALSTOM gasifier benchmark problem as shown in Fig. 1 has five inputs (coal, limestone, air, steam and char extraction) and four outputs (pressure, temperature, bed mass and gas quality) with a high degree of cross coupling between them. Since limestone is used to absorb sulphur in the coal, its flow rate must be set to a fixed ratio of coal flow rate (limestone flow rate $=10 \%$ of coal rate). Therefore, the process can be considered as a $4 \times 4$ process with 4 controlled variables and 4 independently manipulated variables. In addition, there 